Issue 3

Trade for the 99\%

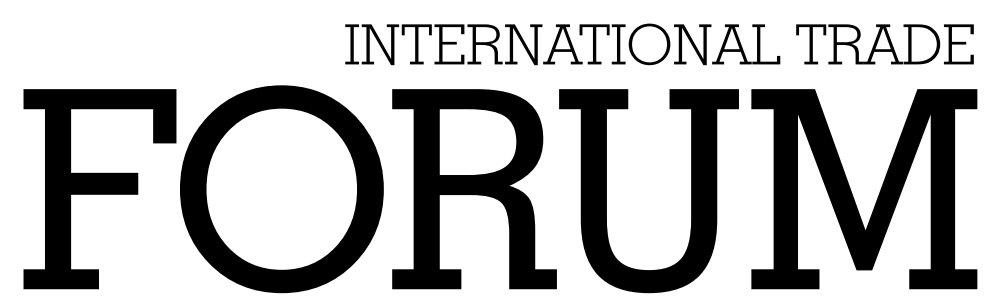

1) 1

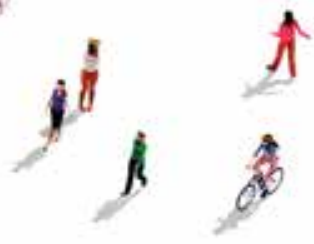

\title{
का
}

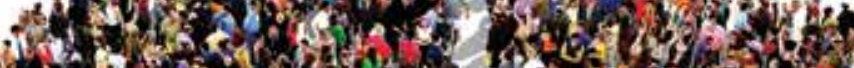

है

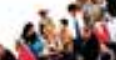
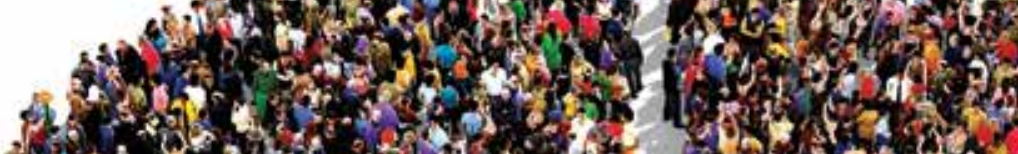

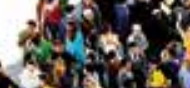<smiles>[AlH2]</smiles>

4

istis

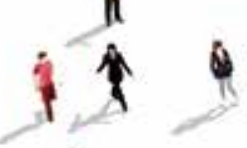

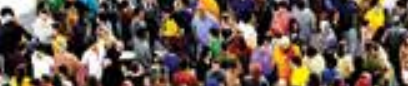

30 s.

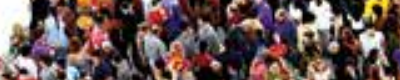

is ${ }^{2}$.

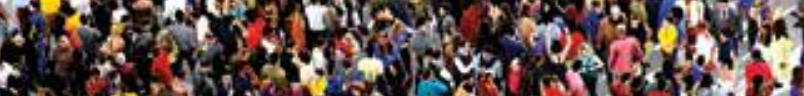

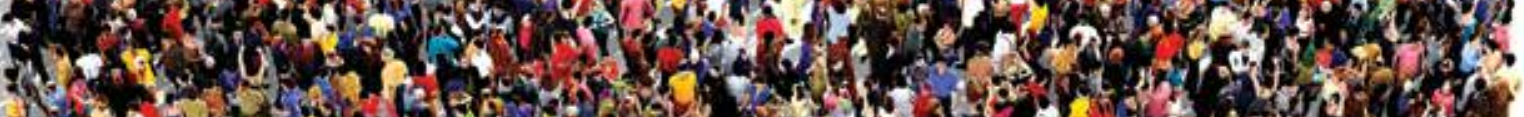

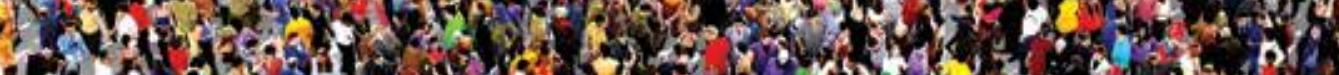

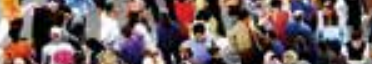

ath

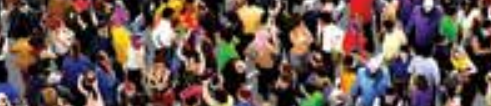

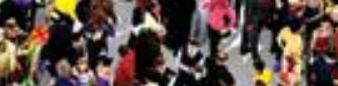

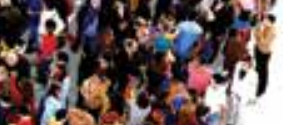

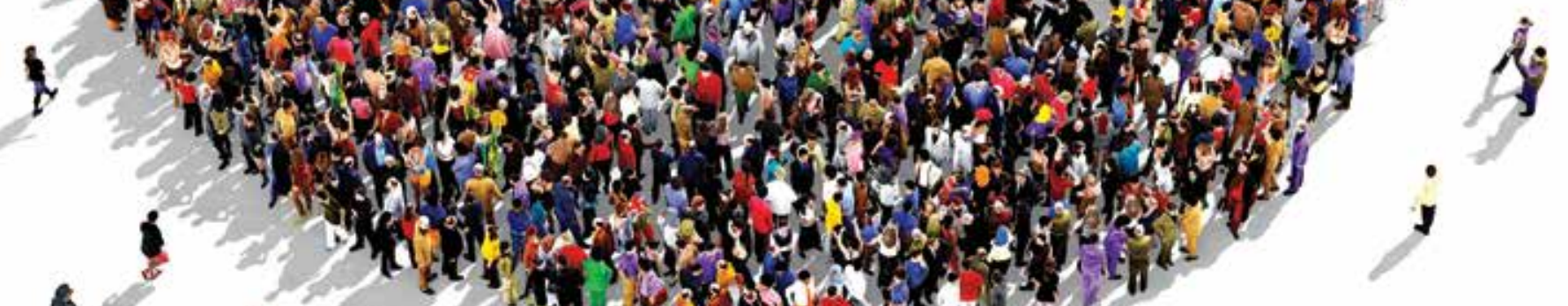

\&

苟

A.

in ${ }^{2}$.
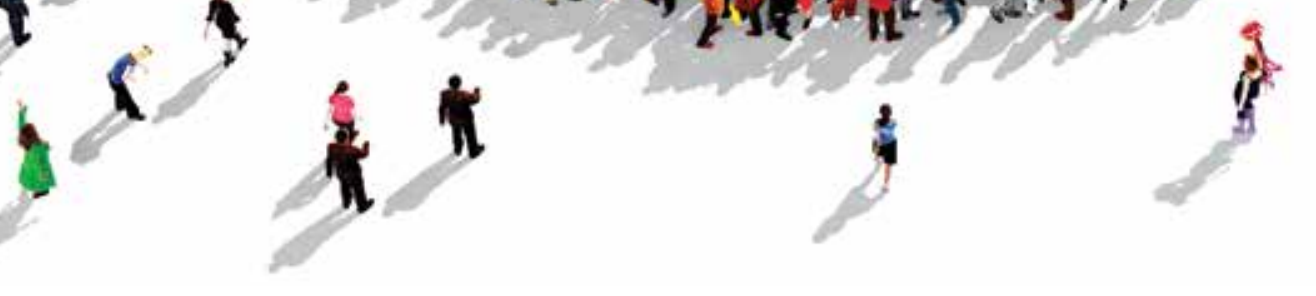

s

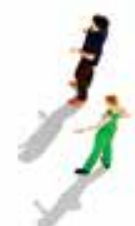




\section{Optimizing the internet for more inclusive trade}

DAVID HAMILTON, Economist, World Trade Organization and CRAIG ATKINSON, Visiting Research Fellow, World Trade Institute

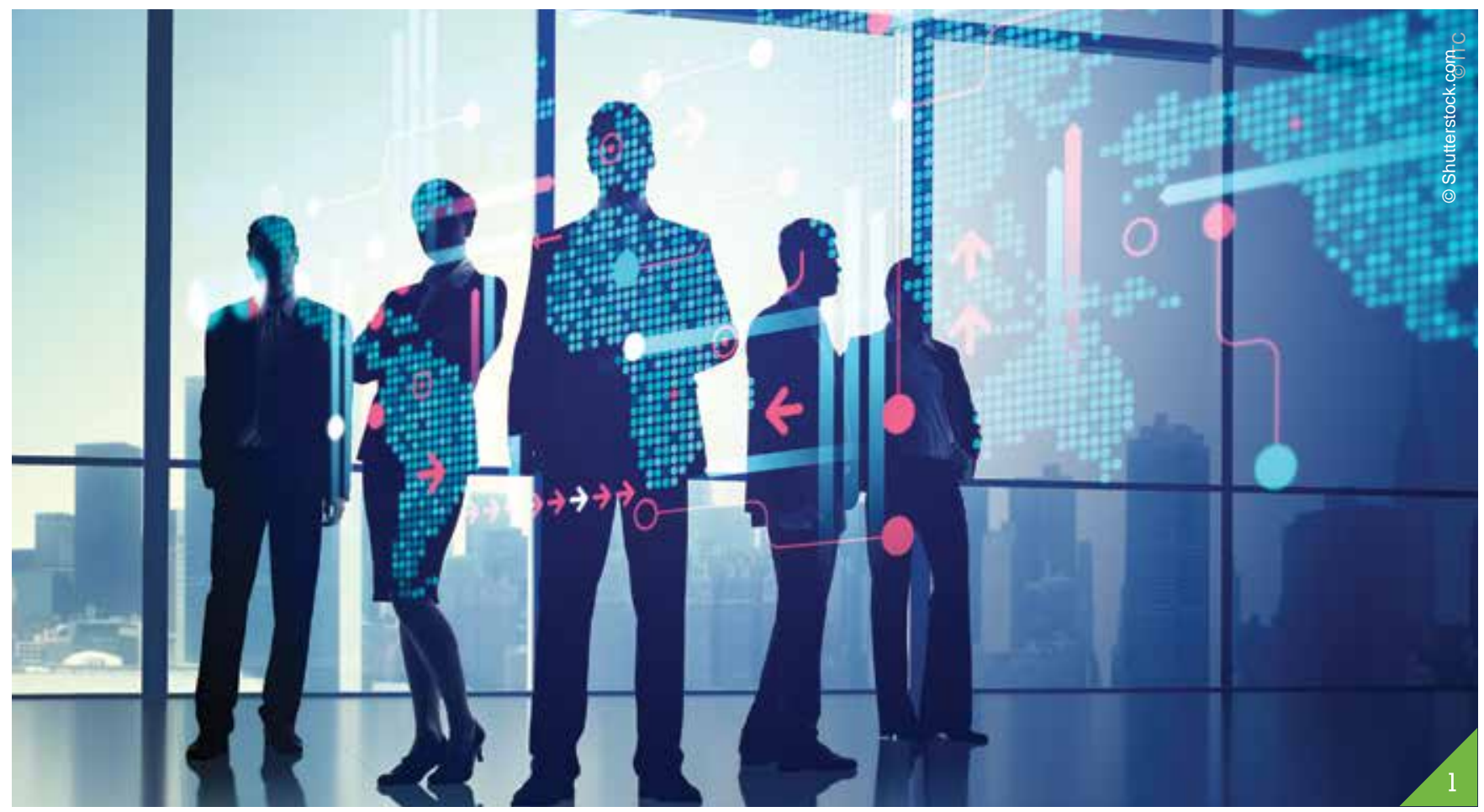

\section{Digital opportunities for inclusive trade can also lower costs for small business}

$\prod$ he internet and e-commerce provide $\perp$ new opportunities for firms of all sizes, located anywhere, to prosper from trade. Likewise, improved access to information represents a rising tide that can lift all boats. The fragmented nature of the current information system for trade is an unintended design flaw that can be addressed through standards, different mediums for policy delivery and the use of appropriate internet-enabled technologies. Improving access to information through trade digitization will enable better global market outcomes, particularly for small firms.
Currently, there are several levels of discussion that refer to making trade more inclusive through digitalization. One level considers access to information. Another emphasizes functionality: simplifying local submission of information, documentation and payments (for example, through a national access window) and its crossborder compliance equivalent. Yet, when considering access to information, it must be determined whether the notion of transparency is merely a legal requirement or if it includes functional outcomes.

Accessing trade information can be a difficult prospect, as disparate systems
Although government's data protection and localization regulations may present a real threat to the future of digitally enabled trade, there are also many opportunities.

provide data inputs of international organizations and governments. While the world is rich in trade-enabling data and information, a lack of functionality originates in its delivery architecture. In this case, valuable and highly skilled human effort is required to navigate the requisite information. This creates costs for consumers, firms and 
While the world is rich in tradeenabling data and information, a lack of functionality originates in its delivery architecture.

governments. However, with technological developments and the emergence of the internet, there are new opportunities to reduce costs associated with asymmetries in the global 'information system' for trade.

Additionally, the advent of a formalized system for collecting national rules and regulations in categories of non-tariff measures (NTMs) has changed how we discuss trade policy. In the past there were only two means of resolving associated issues: harmonization of regulations or mutual recognition. Now, digital technology is enabling opportunities to mitigate the costs associated with NTMs without governments having to change regulations.

While established approaches created the potential for a democratic deficit, new options can avoid this risk (for example, by changing the medium of policy delivery). A market- relevant level of transparency empowers users, reducing uncertainty and informational barriers, and lowers the costs associated with NTMs that hinder or prevent micro, small and medium-sized enterprises (MSMEs) from participating in global markets.

\section{DATA AND NEW BARRIERS}

There is an entire 'supply chain' affecting the data and information that can enable trade. Its efficiency is critical to optimizing market outcomes, including the participation of MSMEs in trade. Its functionality depends on the adequate provision of quality data, information, and minimizing the degree of fragmentation.

Regarding interoperability, it is not enough to provide inputs online: they must be easy to find, understand and process. New initiatives, such as open data and linked data, provide a foundation for the development of new navigation tools. Access to information is not solely about supply or quality: it includes its organization, format and the medium through which it is delivered.

The fragmentation of the information or data supply chain increases costs for MSMEs while greater interoperability decreases them. For example, the World Trade Organization

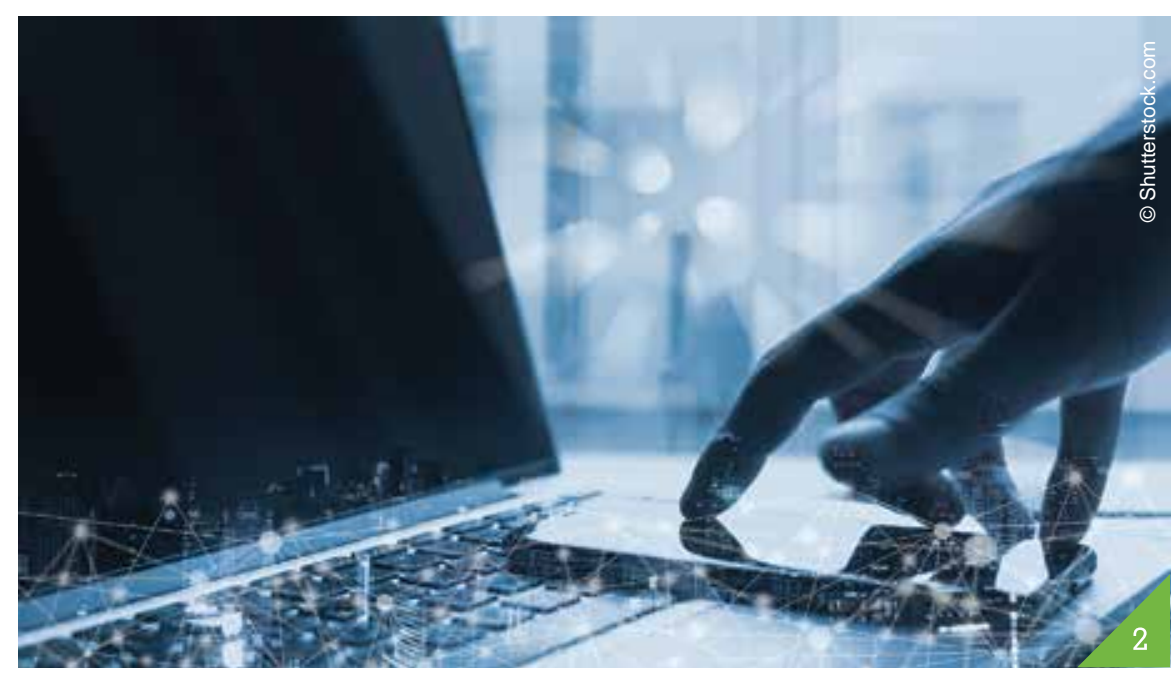

(WTO) Trade Facilitation Agreement requires governments to establish a single access window where traders can submit queries for necessary information. These integrated, internet-enabled, platforms can decrease search times, reduce capability dependencies and lower transaction costs for parties to a cross-border transaction.

Decreasing fragmentation is largely a question of system design. New technologies enable better designs, linkages and market outcomes. Consideration must be given to the set of best practices that will emerge from various initiatives. It is not unreasonable to project that solutions based on better access to information through digital technology will be a major source of best practices for trade facilitation going forward, particularly those associated with implementation of the Trade Facilitation Agreement.

\section{ENTERPRISE SUCCESS}

The speed of information and the governance of data flows can determine the success of firms of all sizes. Several countries are enacting rules and standards for data protection. A regulatory-induced fragmentation of the data supply chain may be seen as a digital trade oversight. Although government's data protection and localization regulations may present a real threat to the future of digitally enabled trade, there are also many opportunities.

Through open-data initiatives and legal-technology policies, both public and private activities are creating potential efficiency gains within the data supply chain for global trade. The 2017 adoption of the United Nations Commission on International
Trade Law (UNCITRAL) Model Law on Electronic Transferable Records (MLETR) suggests that regulations for the use of digital records are becoming mainstreamed. UNCITRAL has adopted the law with the expectation of benefits for speed of information transmission, reuse of record data and a move toward entirely paperless trade. The door also remains open for new multilateral rules at the WTO to clarify obligations related to trade digitization and access to information.

\section{MORE INCLUSIVE TRADE}

Such initiatives are largely to benefit small business. This is particularly relevant when considering the potential for smaller firms to leverage embedded trade-automation technologies in private, business-to-consumer (B2C), e-commerce marketplaces such as Alibaba, Amazon.com and eBay. Different stages of global value chains are also worth considering. There are also significant implications for MSMEs that produce intermediate goods and do not use e-commerce platforms to connect into global value chains.

Although present options for trade digitization to improve access to information are not perfect, they path toward better policy delivery and improved market outcomes. information may represent the best starting point for a discussion on making trade more inclusive with digital technology. $\odot$

1. There is an entire 'supply chain' affecting the data and information that can enable trade.

2. Fragmentation of the information or data supply-chain decreases them. For now, working on access to data and trade increases costs for MSMEs while greater interoperability 